value and zero.

Even though thermal radiation is completely chaotic, an almost perfect interference pattern can be seen in such a set-up for small time delays. However, when the time delay becomes larger than a temperature-dependent quantity called the coherence time, the amplitude of the oscillations with respect to the maximum value quickly decays and the minimum value is no longer zero. Benea-Chelmus et al. observed this behaviour in their experiment at about room temperature (300 kelvin) and found that the coherence time agreed with theoretical predictions.

If the temperature is lowered to a few kelvin, radiation in the terahertz frequency range, which is relevant to such experiments, is suppressed. For example, at $4 \mathrm{~K}$, there are effectively no photons that have frequencies larger than $0.2 \mathrm{THz}$. As a result, in the standard set-up, the maximum amplitude of the oscillations drops to zero and there is no evidence of correlations. Remarkably, Benea-Chelmus and colleagues found that they could still observe correlations in their version of the set-up at $4 \mathrm{~K}$. They interpret these signals as a direct signature of correlations in the electric field of the vacuum.

The authors achieved this feat because of a sophisticated detection scheme. Based on a branch of research known as nonlinear optics, the scheme simultaneously provides two key components that can be pictured in terms of a modified Mach-Zehnder interferometer (Fig. 1b). The first is a pair of elements called temporal gates that 'observe' electric fields in ultrashort time windows - the two observations differ by a time delay. In Benea-Chelmus and colleagues' experiment, these gates are implemented by auxiliary ultrashort pulses of near-infrared light that interact with the vacuum in a nonlinear optical crystal. The second is a pair of detectors that are directly sensitive to the electric fields that have passed through the temporal gates and to the correlations in these fields.

Benea-Chelmus et al. note that the maximum amplitude of the oscillations, although non-zero, was so small that up to one trillion individual detection events at each value of the time delay were required

"The authors
found that they
could still observe
correlations in
their version of
the set-up at
4 kelvin."

for the correlations to be discernible from measurement-induced random fluctuations (shot noise). Such a large number of events took about 3 hours at a repetition rate of $80 \mathrm{MHz}$.

An intriguing aspect of the experiment that deserves further investigation is the effect of the detection procedure, including the use of temporal gates, on the measured fields - a phenomenon called quantum back-action ${ }^{7}$.

Previous work has described how photons materialize in a vacuum for hypothetical, short-lived observers that, because of the finite speed of light, can access information only from restricted regions of space-time, called space-time diamonds ${ }^{8}$. By considering multiple observers in such settings, it has been shown that the photons display quantum correlation called entanglement ${ }^{9}$. The field of quantum optics began with simple experiments that probed amplitude and intensity correlations in optical fields, but now witnesses and applies sophisticated entanglement-based protocols in nascent quantum technologies. Perhaps the authors' results are the first steps towards ultrafast quantum optics that will one day observe and control the entanglement that lies hidden in the spacetime vacuum and in non-trivial ground states of interacting light-matter systems.

Andrey S. Moskalenko is in the Department of Physics, Korea Advanced Institute of Science and Technology, Daejeon 34141, South Korea, and in the Department of Physics, University of Konstanz, Germany. Timothy C. Ralph is at the School of Mathematics and Physics, The University of Queensland, St Lucia, Queensland 4072, Australia.

e-mails: moskalenko@kaist.ac.kr; ralph@physics.uq.edu.au

1. Riek, C. et al. Science 350, 420-423 (2015).

2. Benea-Chelmus, I.-C., Settembrini, F. F., Scalari, G. \& Faist, J. Nature 568, 202-206 (2019).

3. Young, T. Phil. T. R. Soc. Lond. 92, 12-48 (1802).

4. Arndt, M. \& Hornberger, K. Nature Phys. 10 271-277 (2014).

5. Ananthaswamy, A. Through Two Doors at Once: The Elegant Experiment That Captures The Enigma of Our Quantum Reality (Dutton, 2018).

6. Grangier, P., Roger, G. \& Aspect, A. Europhys. Lett. 1, 173-179 (1986).

7. Braginsky, V. B. \& Khalili F. Y. Quantum Measurement (Cambridge Univ. Press, 1992).

8. Martinetti, P. \& Rovelli, C. Class. Quant. Grav. 20 4919-4931 (2003).

9. Su, D. \& Ralph, T. C. Phys. Rev. D 93, 044023 (2016).

\title{
Genetic paradox explained by nonsense
}

\section{Gene mutations that truncate the encoded protein can trigger the expression of} related genes. The discovery of this compensatory response alters our thinking about genetic studies in humans and model organisms. SEE ARTICLE P.193 \& LETTER P.259

\section{MILES F. WILKINSON}

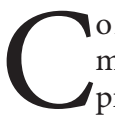
onventional wisdom holds that modifying a gene to make the encoded protein inactive - 'knocking out' the gene - will have more severe effects than merely reducing the gene's expression level. However, there are many cases in which the opposite occurs. In fact, the knockout of a gene sometimes has no discernible impact, whereas the reduction of expression (knockdown) of the same gene causes major defects ${ }^{1}$. Off-target or toxic effects of the reagents used for gene knockdown have sometimes been found to be the culprit ${ }^{2}$, but not always ${ }^{3}$, leaving one to wonder what else could be responsible. El-Brolosy et al. ${ }^{4}$ (page 193) and Ma et al. ${ }^{5}$ (page 259) provide an intriguing solution to this paradox.

The authors identify a molecular mechanism that activates the transcription of genes related to an inactivated gene, thereby compensating for the knockout. The existence of such a genetic compensation response was initially suggested by earlier studies, most notably the discovery ${ }^{3}$ that knockout of the egfl7 gene in zebrafish upregulates the expression of genes that encode proteins related to those encoded by egfl7. This upregulatory response was triggered only by mutation of egfl7, and not by egfl7 knockdown, thereby explaining why knockdown caused biological defects, whereas knockout largely did not. Similar observations have been made for other genes $^{3,6}$, suggesting the existence of a general compensatory mechanism.

How does the compensatory mechanism work? By studying the effects of a variety of mutations in zebrafish embryos, both El-Brolosy et al. and Ma et al. found that the upregulation of compensatory genes is specifically triggered by mutations that generate short nucleotide sequences known as premature termination codons (PTCs). These sequences - also known as nonsense codons - signal the early cessation of the translation of messenger RNAs into proteins. Thus, an apt name for this upregulatory response is nonsense-induced transcriptional compensation (NITC).

The role of PTCs in NITC suggested the involvement of an RNA-degradation pathway called nonsense-mediated RNA decay (NMD), because NMD is also triggered by PTCs ${ }^{7}$. In support of this idea, El-Brolosy and colleagues 


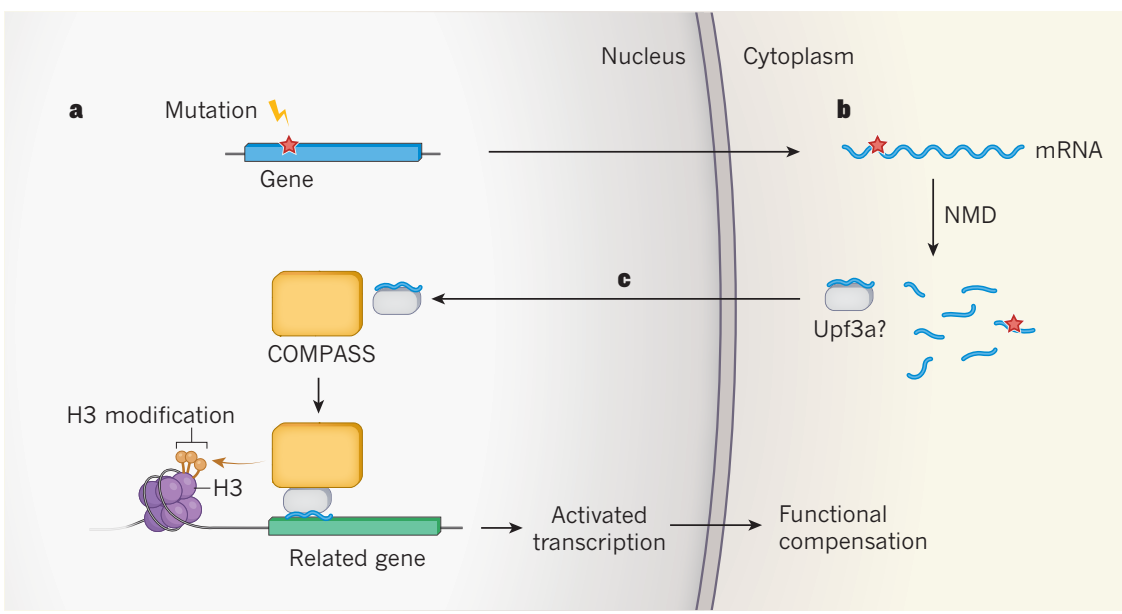

Figure 1 | Proposed mechanism for mutation-induced biological compensation. El-Brolosy et al. ${ }^{4}$ and $\mathrm{Ma}$ et al. ${ }^{5}$ report that mutations that lead to the production of truncated, often non-functional proteins indirectly activate the expression of related genes, to provide functional compensation. a, The mutations observed to trigger this process generate DNA sequences (red star) that lead to the production of messenger RNA molecules for which translation is prematurely terminated. $\mathbf{b}$, These sequences also trigger mRNA degradation by a process called nonsense-mediated RNA decay (NMD), which both studies implicate in the compensatory response. $\mathbf{c}$, El-Brolosy et al. propose that mRNA fragments generated by NMD bind selectively to complementary nucleotide sequences in related genes, and carry unknown regulatory factors to induce transcription. One of these factors might be Upf3a, an NMD factor that $\mathrm{Ma}$ et al. show is crucial for the transcriptional compensatory mechanism. They also find that Upf3a interacts with COMPASS - a protein complex that activates gene transcription by modifying one of the histone proteins (H3) with which genes are packaged in the nucleus. Both groups find that COMPASS is integral to the genetic compensatory response.

observed that a protein called Upf1 - one of the main factors involved in NMD - is required for NITC. Further support came from $\mathrm{Ma}$ and co-workers' discovery that PTCs that do not trigger NMD also failed to elicit NITC.

The two papers provide several lines of evidence suggesting that the agent that triggers NITC is the PTC-bearing mRNA generated from a mutant gene (Fig. 1). For example, both groups found that NITC is largely prevented when transcription of the mutant gene is compromised. As evidence that the PTC-bearing mRNA must be degraded by NMD to elicit NITC, El-Brolosy et al. found that injection of 'uncapped' mRNA, which is inherently unstable, into zebrafish embryos elicited NITC, whereas 'capped' (stable) mRNA did not.

Both groups found that NITC is mediated by increased transcription of genes that are ancestrally related to the mutant gene. The increased transcription of these gene paralogues was accompanied by increased occupancy of the genes' promoter regions by H3K4me3 - a transcription-inducing form ${ }^{8}$ of a DNA-binding protein called histone $\mathrm{H} 3$. The two groups went on to find that NITC depends on the COMPASS protein complex, which catalyses ${ }^{8}$ the formation of $\mathrm{H} 3 \mathrm{~K} 4 \mathrm{me} 3$, thereby providing further evidence that this H3 modification has a role in the increased transcription of the gene paralogues.

How could a decaying mRNA specifically trigger transcriptional upregulation of genes similar to itself, but not of unrelated genes? On the basis of the finding that some factors required for the decay of RNA in the cytoplasm also 'moonlight' as transcriptional regulators in the nucleus', El-Brolosy et al. posit a model in which cytoplasmic-RNA decay factors associated with PTC-bearing mRNA travel to the nucleus and recruit histone modifiers to activate the transcription of related genes. Specificity is provided by the RNA fragments generated by NMD, which bind to complementary nucleotide sequences in the gene paralogues (Fig. 1). This model also predicts that heterozygotic zebrafish, which have both a mutant and a wild-type copy of a gene, will upregulate the expression of the wild-type gene. Indeed, both research groups found that this is the case.

A key discrepancy between the two papers is that El-Brolosy et al. found that knockout of the $u p f 1$ gene prevented NITC, whereas Ma et al. observed that NITC was not impaired by knockdown of Upf1, nor by knockdown of the NMD factors Upf2 and Upf3b. It remains to be seen whether this apparent discrepancy results from the use of different approaches for perturbing Upf1, from introducing PTCs in different genes, or from other factors.

An intriguing discovery made by Ma et al. is that NITC requires the Upf3a protein, an enigmatic NMD factor that was previously shown ${ }^{10}$ to either repress or modestly promote NMD, depending on the mRNA targeted for degradation. The authors found that Upf3a interacts with several components of the COMPASS complex, and increases H3K4me3 occupancy at the promoter regions of upregulated paralogue genes. These findings led $\mathrm{Ma}$ et al. to propose that Upf3a recruits the COMPASS complex to gene-paralogue promoters to generate the $\mathrm{H} 3 \mathrm{~K} 4 \mathrm{me} 3$ mark responsible for activating gene transcription.

The discovery of NITC will probably alter how genetic studies are interpreted and conducted in the future. For example, the unexpected finding that healthy humans typically have several loss-of-function mutations ${ }^{11}$ can now potentially be explained by compensation conferred by NITC. When constructing genetically engineered model organisms, it will be important to make mutants that do not trigger NITC, to avoid biological defects being masked. Indeed, NITC might explain why many knockouts produce few biological changes in mice, whereas chemical agents that block or activate the same targets have profound effects ${ }^{12,13}$.

El-Brolosy et al. demonstrated that NITC provides protective compensation not only in zebrafish embryos, but also in mouse cell lines. NITC's reach might extend much further than this, given that gene knockouts in diverse organisms have been shown to produce less-severe defects than corresponding gene knockdowns ${ }^{1}$. How broadly does NITC act in a given organism? El-Brolosy et al. and Ma et al. together identified eight zebrafish genes and four mouse genes that, when mutated, trigger the NITC mechanism, but it remains unclear whether this is a property of most genes. It is also unknown what dictates NITC's strength and specificity. Both research groups identified some specific mutant genes and mutations that trigger NITC only weakly, implying that there are factors yet to be understood that modulate NITC.

NITC adds to the arsenal of mechanisms that confer robustness on organisms in response to mutations. Indeed, NITC provides hope even when a gene has been completely inactivated by mutation. In the words of the writer Henry David Thoreau: "If we will be quiet and ready enough, we shall find compensation in every disappointment." -

Miles F. Wilkinson is in the Department of Obstetrics, Gynecology, and Reproductive Sciences, University of California, San Diego, La Jolla, California 92093, USA.

e-mail:mfwilkinson@ucsd.edu

1. El-Brolosy, M. A. \& Stainier, D. Y. R. PLoS Genet. 13, e1006780 (2017).

2. Kok, F. O. et al. Dev. Cell 32, 97-108 (2015).

3. Rossi, A. et al. Nature 524, 230-233 (2015).

4. El-Brolosy, M. A. et al. Nature 568, 193-197 (2019).

5. Ma, Z. et al. Nature $568,259-263$ (2019).

6. Zhu, P. et al. J. Genet. Genom. 44, 553-556 (2017).

7. Popp, M. W.-L. \& Maquat, L. E. Annu. Rev. Genet. 47, 139-165 (2013).

8. Howe, F. S., Fischl, H., Murray, S. C. \& Mellor, J. BioEssays 39, 1600095 (2016).

9. Haimovich. G. et al. Cell 153, 1000-1011 (2013).

10.Shum, E. Y. et al. Cell 165, 382-395 (2016).

11. MacArthur, D. G. et al. Science 335, 823-828 (2012).

12.Brooks, P. C. et al. Cell 79, 1157-1164 (1994).

13.Bader, B. L., Rayburn, H., Crowley, D. \& Hynes, R. O. Cell 95, 507-519 (1998).

This article was published online on 3 April 2019. 